\title{
Análise do uso de cateter central de inserção periférica em Unidade de Cuidado Intensivo Neonatal
}

\author{
Peripherally inserted central catheter at the Neonatal Intensive Care Unit
}

\author{
Análisis del uso de catéter central de inserción periférica en Unidad de Cuidado Intensivo Neonatal
}

Patrícia Fernanda de Almeida Cabral', Patrícia Kuerten Rocha², Sayonara de Fátima Faria Barbosa3,

Grace Terezinha Marcon Dal Sasso ${ }^{4}$, Rodrigo Otávio Moretti-Pires ${ }^{5}$

\footnotetext{
${ }^{1}$ Enfermeira. Discente do Programa de Pós-Graduação em Enfermagem do Departamento de Enfermagem (NFR) da Universidade Federal de Santa Catarina (UFSC) Florianópolis, SC, Brasil. E-mail: patifac@yahoo.com.br.

2 Enfermeira, Doutora em Enfermagem. Professora Adjunta do NFR/UFSC. Florianópolis, SC, Brasil. E-mail: patriciarocha@ccs.ufsc.br.

${ }^{3}$ Enfermeira, Doutora em Ciências da Saúde. Professora Adjunta do NFR/UFSC. Florianópolis, SC, Brasil. E-mail: sayonara@ccs.ufsc.br.

${ }^{4}$ Enfermeira, Doutora em Enfermagem. Professora Adjunta II do NFR/UFSC. Florianópolis, SC, Brasil. E-mail: grace@ccs.ufsc.br.

${ }^{5}$ Enfermeiro, Doutor em Enfermagem Psiquiátrica. Professor Adjunto do Departamento de Saúde Pública da UFSC. Florianópolis, SC, Brasil. E-mail: rodrigomoretti@ccs.ufsc.br.
}

\section{RESUMO}

O objetivo deste estudo foi traçar o perfil dos neonatos que fizeram uso de PICC. Trata-se de pesquisa retrospectiva, descritiva, com amostra final de 163 neonatos. Os dados foram coletados dos prontuários de neonatos internados em uma Unidade de Cuidado Intensivo Neonatal (UCIN) de um Hospital Universitário em Florianópolis-SC, entre os anos de 2004 e 2009. A maioria dos neonatos era do sexo masculino, de raça branca e com diagnóstico médico de prematuridade. A maioria das punções foi realizada em membros superiores e a necessidade de nutrição parenteral foi o motivo predominante. Os cateteres foram sacados, na maioria, pela finalização do tratamento, e as pontas, enviadas para cultura. Verificamos que o PICC preveniu repetidas punções, pois a maioria dos neonatos apresentava fragilidade venosa pela prematuridade. O patógeno encontrado na cultura, Staphylococcus sp., faz parte da microbiota da pele, sugerindo assim, maior precaução na inserção e manutenção do PICC.

Descritores: Recém-Nascido; Unidades de Terapia Intensiva Neonatal; Enfermagem Neonatal; Infecções relacionadas a Cateter.

\section{ABSTRACT}

The objective of this study was to outline the profile of the newborns who used PICC. This retrospective, descriptive study was performed with a sample of 163 newborns. The data were collected from the records of newborn inpatients at a Neonatal Intensive Care Unit (NICU) of a University Hospital in Florianópolis, Santa Catarina, Brazil, between the years of 2004 and 2009. Most newborns were male, of white ethnicity and medically diagnosed with prematurity. Most punctures were performed on the upper limbs and were due to the need for parenteral nutrition. Most catheters were removed only at the end of treatment, and the tips were sent for culture. PICC avoided repeated punctures, as most newborns showed venous frailty due to their prematurity. The pathogen found in the culture, Staphylococcus sp., is part of the skin microbiota, which suggests a need for greater precaution in the placement and maintenance of the PICC.

Descriptors: Infant, Newborn; Intensive Care Units, Neonatal; Neonatal Nursing; Catheter-Related Infections.

\section{RESUMEN}

Se objetivó determinar el perfil de neonatos en los que se utilizó PICC. Se trató de un estudio retrospectivo, descriptivo, con muestra final de 163 neonatos. Datos recogidos de historias clínicas de neonatos internados en Unidad de Cuidado Intensivo Neonatal (UCIN) de un Hospital Universitario de Florianópolis-SC, entre 2004 y 2009. La mayoría de los neonatos era de sexo masculino, raza blanca, con diagnóstico médico de prematuridad. La mayoría de las punciones fue efectuada en miembros superiores y la necesidad de nutrición parenteral fue el motivo predominante. Los catéteres fueron extraídos, mayoritariamente, por finalización del tratamiento; y las puntas, enviadas para cultivo. Verificamos que el PICC previno repetidas punciones; la mayoría de los neonatos presentaba fragilidad venosa por la prematuridad. El patógeno encontrado en el cultivo, Staphylococcus sp., forma parte de la microbiota de la piel, sugiriendo esto mayores precauciones en la inserción y mantenimiento del PICC.

Descriptores: Recién Nacido; Unidades de Cuidado Intensivo Neonatal; Enfermería Neonatal; Infecciones Relacionadas con Catéteres. 


\section{INTRODUÇÃO}

O acesso vascular é frequentemente exigido em neonatos hospitalizados em unidades de cuidados intensivos devido a uma série de indicações clínicas. Com os avanços tecnológicos em cateteres vasculares, uma variedade de opções está disponível para a equipe que cuida destes bebês.

A decisão do dispositivo a ser utilizado para a obtenção de um acesso vascular pode ser um grande desafio. Fatores tais como idade, tamanho, disponibilidade do local para a punção, tipo de medicações ou fluidos a serem administrados, duração previsível de utilização do cateter, como também as possíveis complicações que podem ocorrer, influenciam na tomada de decisão da equipe sobre qual dispositivo utilizar $^{(1-3)}$.

As indicações para a obtenção de acesso vascular em neonatos são numerosas. Em geral, os acessos vasculares podem ser essencialmente divididos em duas grandes categorias: venoso central e periférico. Os acessos venosos periféricos são seguros para administrar medicações, fluidos intravasculares e para a coleta de amostras de sangue, porém, nota-se que esse tipo de punção em neonatos permanece pérvia por pouco tempo. Assim, quando há necessidade de um tratamento por longo prazo ou de acesso venoso central, pode-se optar por utilizar um Cateter Central de Inserção Periférica (PICC), pois este apresenta algumas vantagens na neonatologia, sendo uma delas a diminuição do número de punções repetidas e, por consequência, o risco de infecção. Além disto, este tipo de cateter possibilita infusão de antibióticos, quimioterapia, nutrição parenteral total e substâncias vesicantes ${ }^{(3-6)}$.

Os PICCs a princípio foram desenvolvidos para o uso em neonatos, devido ao pequeno diâmetro do cateter e a flexibilidade do material. Tais cateteres tornaram-se populares na década de 1980, principalmente nos Estados Unidos, quando pacientes em cuidados domiciliares necessitavam de acessos venosos centrais, e desde então, a sua utilização tem crescido gradualmente. Esse sucesso se dá pela redução potencial de complicações em comparação a outros tipos de cateteres centrais, além de ser um procedimento que enfermeiros treinados podem realizar, conforme previsto na Resolução n² 258/2001 do Conselho Federal de Enfermagem ${ }^{(1-3)}$.

Os PICCs são facilmente inseridos sob condições estritamente estéreis, compostos de materiais biocompatíveis e com uma variedade de tamanhos, estando disponíveis no mercado com um ou múltiplos lumens $^{(1,3)}$. Oferecem muitas vantagens para a população neonatal, incluindo baixa dos custos globais e riscos em comparação com os métodos cirúrgicos vasculares de acesso. Ainda, são menos prováveis de desalojar, e podem ser facilmente removidos quando o tratamento é interrompido ou quando complicações, tais como infecção ou flebite ocorrem ${ }^{(1)}$.

Devido ao seu pequeno diâmetro, uma desvantagem de usar o PICC é o aumento da taxa de oclusão. Além disto, também não é recomendado para coleta de sangue, devido à possibilidade do cateter colabar durante a aspiração ${ }^{(1,7-9)}$.

A presença de sinais e sintomas no local da inserção do cateter como eritema, edema e presença de secreção purulenta podem representar infecção, seja esta local ou sistêmica. A infecção local geralmente exige remoção do dispositivo e tratamento com antibióticos intravenosos, conforme necessário. Como regra, a maioria das infecções relacionadas com cateter pode ser tratada com sucesso com antibióticos intravenosos sem a remoção do mesmo. No entanto, quando estas infecções são causadas por fungos e bactérias gram-negativas o mais adequado é o tratamento com medicamentos e a retirada do cateter ${ }^{(10)}$.

A prevenção da infecção do cateter é um fator prioritário aos profissionais de saúde, sendo necessária uma rigorosa atenção quanto à lavagem de mãos, as técnicas assépticas para manusear o cateter, e minucioso cuidado do local de inserção do dispositivo. Há uma diminuição do número de infecções quando cuidados específicos com o cateter são estabelecidos por meio do uso de protocolos e profissionais bem treinados ${ }^{(8,10-12)}$.

A partir disto, o objetivo desta pesquisa foi verificar o perfil dos neonatos admitidos em uma Unidade de Cuidados Intensivos Neonatais que utilizaram PICC.

\section{METODOLOGIA}

Consistiu em um estudo retrospectivo, descritivo, realizado em uma Unidade de Cuidado Intensivo Neonatal (UCIN), em um Hospital Universitário de Santa Catarina. Os dados foram coletados a partir dos prontuários dos neonatos que fizeram uso de PICC no período de 2004 a 2009. A escolha deste período deu-se pelo início da utilização do PICC nesta unidade. A pesquisa foi realizada 
entre os meses de maio de 2010 a fevereiro de 2011.

A população foi constituída de todos os neonatos que fizeram uso de PICC, ou seja, um total de 233 recémnascidos no período citado. Os critérios de inclusão foram: ser neonato admitido em UCIN no período da coleta e ter feito uso de PICC. Assim, a amostra foi constituída de 163 neonatos, pois houve uma perda de 70 crianças devido ao registro incorreto quanto ao uso do cateter ou número do registro hospitalar.

As variáveis estudadas foram sexo, idade gestacional (IG), peso ao nascimento (Pn), tipo de parto, Apgar no primeiro e no quinto minutos, raça, diagnóstico médico, motivo de inserção do PICC, local de inserção, terapia utilizada, tempo de permanência do cateter, motivo da retirada e resultado da cultura da ponta do cateter.

O estudo consistiu em três etapas. A primeira etapa teve como objetivo selecionar a amostra do estudo. Assim, foram identificados por meio de um levantamento do censo da Comissão de Controle de Infecção Hospitalar (CCIH) em questão, todos os neonatos que utilizaram PICC no período de janeiro de 2004 a dezembro de 2009. Na segunda etapa foi elaborado um instrumento para coleta de dados, sendo que foi realizado um pré-teste do mesmo em 10 prontuários. Neste momento o instrumento foi aplicado nos prontuários dos neonatos no Serviço do Prontuário do Paciente. E, a terceira etapa teve como objetivo analisar os dados encontrados. Os dados obtidos foram tabulados em planilha utilizando a ferramenta Microsoft Excel ${ }^{\circledR}$ (versão 2003), e analisados de forma não probabilística, por meio do sistema SESTATNET ${ }^{\circledR}$ da Universidade Federal de Santa Catarina, utilizando estatística descritiva contendo frequência absoluta e relativa, médias e desvio padrão.

As normas regulamentadoras da pesquisa com seres humanos, ditadas na Resolução 196/96 foram respeitadas durante todo o seu processo, como também, a pesquisa passou pela análise do Comitê de Ética da Universidade Federal de Santa Catarina (protocolo número 388/08).

\section{RESULTADOS}

Verificamos um total de 163 crianças que utilizaram PICC no período do estudo, sendo que o número de cateteres inseridos nas mesmas foi de 186 PICC's. Assim, podemos constatar que em 18 (11\%) neonatos foram utilizados dois ou mais PICC's.

Houve um crescimento gradual no número de neonatos que receberam PICC durante os anos de 2004 a 2009. Em 2004, sete (04\%) neonatos utilizaram PICC, em 2005 somou 27 (14\%), em 2006 foram 28 (15\%), em 2007 aumentou para 34 (19\%), em 2008 foram 43 (23\%), e em 2009 o resultado foi de 47 (25\%) procedimentos realizados.

Quanto à caracterização dos neonatos que realizaram o procedimento (Tabela 1) verifica-se que os que fizeram uso de PICC eram na sua maioria do sexo masculino somando 95 (58\%) das crianças, predominantemente da raça branca (87\%), com idade gestacional entre 25 a 30 semanas (55\%), que receberam escore Apgar de 8 no primeiro e no quinto minuto de vida, apresentaram peso entre 1101 a $1700 \mathrm{~g}$ ao nascer (47\%), nasceram de parto cesáreo (61\%), e receberam diagnóstico de prematuridade na ocasião da internação na unidade neonatal. A indicação do uso do PICC foi devido a necessidade de nutrição parenteral (43\%). 
Tabela 1: Características dos neonatos que utilizaram PICC ( $n=163)$. Florianópolis, SC, 2011.

\begin{tabular}{|c|c|c|}
\hline Variáveis & $\mathbf{f}$ & $\%$ \\
\hline \multicolumn{3}{|l|}{ Idade Gestacional ao Nascimento } \\
\hline$\leq 24$ semanas & 03 & 02 \\
\hline 25 - 30 semanas & 90 & 55 \\
\hline $31-35$ semanas & 49 & 30 \\
\hline $36-42$ semanas & 16 & 10 \\
\hline Sem registro & 05 & 03 \\
\hline Peso ao Nascimento & 20 & 11 \\
\hline و500g-1100 & 63 & 39 \\
\hline و1700 - و1101 & 76 & 47 \\
\hline و & 13 & 08 \\
\hline 2301g-2900و & 04 & 02 \\
\hline 2901g-3500g & 06 & 04 \\
\hline 3501g-4100g & 01 & 01 \\
\hline \multicolumn{3}{|l|}{ Diagnóstico } \\
\hline Prematuridade & 76 & 46 \\
\hline Prematuridade e baixo peso & 28 & 17 \\
\hline Prematuridade e desconforto respiratório & 32 & 20 \\
\hline Prematuridade, baixo peso e desconforto respiratório & 05 & 03 \\
\hline Infecções perinatais & 19 & 12 \\
\hline Outros & 03 & 02 \\
\hline \multicolumn{3}{|l|}{ Indicação do uso do cateter } \\
\hline Fragilidade Venosa & 13 & 08 \\
\hline Terapia Endovenosa & 42 & 26 \\
\hline Antibioticoterapia & 20 & 12 \\
\hline Nutrição Parenteral Total/antibioticoterapia & 70 & 43 \\
\hline Outros & 16 & 10 \\
\hline Sem registro & 02 & 01 \\
\hline \multicolumn{3}{|l|}{ Dias de vida quando da inserção } \\
\hline $01-05$ dias & 140 & 85 \\
\hline $06-10$ dias & 20 & 12 \\
\hline$\geq 11$ dias & 05 & 03 \\
\hline
\end{tabular}

Os cateteres (Tabela 2) tinham na sua maioria calibre de 1,9 Fr, sendo que o local predominante de inserção foi os membros superiores com 164 (88\%) das punções. As veias escolhidas com maior frequência e registradas no prontuário foram a basílica, a mediana cubital, a radial e a cefálica. Cabe ressaltar que geralmente estava registrado que a punção tinha sido realizada no membro superior, porém não estava especificada a veia escolhida para o procedimento. A terapêutica mais utilizada foi a nutrição parenteral total e antibiótico terapia. Não havia nos prontuários registro quanto aos curativos (frequência e material utilizado) realizados no local de inserção do cateter; comprimento externo do cateter; número de tentativas e intercorrências no momento da passagem; soluções utilizadas e número de lavagens do cateter por dia; e, forma de desobstrução do cateter. Os cateteres permaneceram, em sua maioria, por um período de seis a quinze dias, sendo retirados principalmente devido à finalização do tratamento em 110 (59\%) dos casos, por perda do acesso em 46 (25\%) do total, em 21 (11\%) por presença de sinais flogísticos e em nove (5\%) por outros motivos. 
Tabela 2: Características dos PICCs ( $n=186)$. Florianópolis, SC, 2011.

\begin{tabular}{|c|c|c|}
\hline Variáveis & $\mathbf{f}$ & $\%$ \\
\hline \multicolumn{3}{|l|}{ Calibre } \\
\hline $1,9 \mathrm{Fr}$ & 171 & 92 \\
\hline $2,0 \mathrm{Fr}$ & 15 & 08 \\
\hline \multicolumn{3}{|l|}{ Localização Rx } \\
\hline Central & 46 & 25 \\
\hline Periférico & 48 & 26 \\
\hline Local impróprio & 48 & 26 \\
\hline Sem registro & 44 & 23 \\
\hline \multicolumn{3}{|l|}{ Terapêutica utilizada } \\
\hline Nutrição parenteral total & 33 & 18 \\
\hline Nutrição parenteral total/antibioticoterapia & 111 & 60 \\
\hline Antibioticoterapia & 24 & 13 \\
\hline Nutrição parenteral total/antibioticoterapia/antifúngicos & 08 & 04 \\
\hline Outras medicações & 08 & 04 \\
\hline Sem registro & 02 & 01 \\
\hline \multicolumn{3}{|l|}{ Tempo de Permanência } \\
\hline $01-05$ dias & 22 & 12 \\
\hline $06-10$ dias & 65 & 35 \\
\hline $10-15$ dias & 65 & 35 \\
\hline $16-20$ dias & 24 & 13 \\
\hline $21-25$ dias & 02 & 01 \\
\hline $25-30$ dias & 02 & 01 \\
\hline Sem registro & 06 & 03 \\
\hline
\end{tabular}

Não havia descrito no prontuário dos neonatos sinais flogísticos no local da inserção do cateter, porém verificamos que dos 186 cateteres inseridos, 123 (77\%) tiveram as pontas enviadas para a realização de cultura
(Tabela 3). Destes, 13 (11\%) tiveram resultado de cultura positivo, sendo o agente etiológico predominante o Staphylococcus sp $(53,8 \%)$.

Tabela 3: Cultura das pontas dos cateteres ( $n=186)$. Florianópolis, SC, 2011.

\begin{tabular}{|c|c|c|}
\hline Variáveis & $\mathbf{f}$ & $\%$ \\
\hline \multicolumn{3}{|l|}{ Cateteres } \\
\hline Enviados para cultura & 123 & 77 \\
\hline Não enviados para cultura & 63 & 23 \\
\hline \multicolumn{3}{|l|}{ Resultado das culturas } \\
\hline Cultura com resultado negativo & 110 & 89 \\
\hline Cultura com resultado positivo & 13 & 11 \\
\hline \multicolumn{3}{|l|}{ Agente etiológico } \\
\hline Staphylococcus coagulase negativo & 07 & 53 \\
\hline Staphylococcus aureus & 03 & 23 \\
\hline Streptococcus sp & 01 & 08 \\
\hline Klebsiella pneumoniae & 01 & 08 \\
\hline Sem agente etiológico registrado & 01 & 08 \\
\hline
\end{tabular}

\section{DISCUSSÃO}

O crescimento gradual no número de PICC's instalados na instituição no período estudado denota a aprovação do PICC pela equipe de saúde, e consequente capacitação dos componentes da unidade para tal. O objetivo de se implantar esta técnica na unidade foi de diminuir o número recorrente de punção periférica e também, a promoção de uma via de acesso central com 
menos riscos do que a dissecção venosa profunda. Outro fator relevante que se pode constatar com o aumento do número de PICCs implantados, é a convergência entre a equipe médica e de enfermagem no intuito de garantir a segurança do paciente, já que estes dispositivos intravenosos propiciam um número menor de infecção e intercorrências ${ }^{(1,13)}$.

A caracterização dos neonatos quanto à predominância do sexo masculino, do parto cesáreo, do baixo peso (1101 a 1700g) e a indicação de instalação do PICC para o recebimento de Nutrição Parenteral Total e terapia endovenosa estão relacionados diretamente com as complicações da prematuridade, principalmente devido à dificuldade de sucção, síndrome do desconforto respiratório ou doença da membrana hialina ${ }^{(14-15)}$.

Cabe ressaltar que na Neonatologia um dos diagnósticos predominantes é a prematuridade, convergindo assim com o estudo realizado, onde o diagnóstico médico inicial foi a prematuridade isolada ou associada a baixo peso ou desconforto respiratório. Devido a este fator e a provável hospitalização prolongada destes neonatos há a indicação do uso do $\operatorname{PICC}^{(14)}$.

Os PICCs tinham em quase sua totalidade o diâmetro de 1,9 Fr, e a inserção dos mesmos foi predominante nos membros superiores, sendo isto considerado como ideal para o uso desse cateter em recém-nascidos de baixo peso(16). Porém, denota-se a falta de registro quanto a veia utilizada para o acesso.

A localização da ponta do cateter pelo RX nos mostrou a locação adequada em $25 \%$ dos PICCs inseridos, ou seja, somente 46 dos 186 PICCs estavam centrais. Entretanto, precisamos levar em consideração que não havia registro da localização da ponta do cateter em $23 \%$ dos registros. Ainda, em $52 \%$ dos registros os cateteres estavam posicionados perifericamente ou 0 posicionamento estava impróprio, ressaltando a importância de se realizar um treinamento com a equipe. A literatura nos apresenta que a localização ideal para o
PICC é na veia cava superior ${ }^{(17)}$.

O tempo de permanência do PICC, observado na maioria dos casos, foi de seis a 15 dias, sendo que o mínimo foi de um dia e o máximo de 30 dias. O principal motivo de retirada foi o término do tratamento. Estes dados são condizentes com estudos realizados em outras UCIN que demonstram uma média de tempo de uso de cateter de oito a nove dias, ou ainda 12,9 dias, sendo o motivo da retirada também a finalização do tratamento ${ }^{(18)}$.

O resultado das culturas das pontas dos cateteres evidenciaram que o agente predominante foi 0 Staphylococcus coagulase negativo com 53\% dos 13 cateteres infectados. Este microorganismo que pertence a microbiota da pele, se torna muitas vezes um agente oportunista no caso de recém-nascidos, principalmente com o uso de cateter venoso central, da nutrição parenteral total e do manuseio do hub. Além da origem endógena, da pele e mucosa do neonato, pode ter origem exógena, ou seja, do meio hospitalar ou das mãos dos profissionais de saúde ${ }^{(19-20)}$.

\section{CONSIDERAÇÕES FINAIS}

O estudo constatou a maior utilização do PICC em recém-nascidos da UCIN estudada. Evidenciou-se a falta de registro de informações relevantes, tais como as características do cateter, o local de inserção, as trocas de curativo, o número de tentativas de punção, as intercorrências no momento da progressão do cateter, as soluções utilizadas e o número de lavagens do cateter por dia, a forma de desobstrução do cateter, entre outros. Isto demonstra a necessidade da implantação de um protocolo de passagem e manuseio do PICC.

Além disto, verificamos que as pontas de cateteres foram localizadas em locais impróprios e cateteres foram sacados com apenas um dia de uso, sugerindo a necessidade de se realizar qualificações e treinamentos com a equipe.

Management practices of peripherally Inserted central Catheter at a neonatal unit. Rev. bras. enferm. 2011;64(6):997-1002.

4. Cheung E, Baerlocher $M$, Asch M, Myers A. Venous access: a pratical review for 2009. Can Fam Physician. 2009;55(5):494-6. 5. Xia B, Tang J, Xiong Y, XH L, DZ M. Peripherally inserted central catheters and the incidence of candidal sepsis in VLBW and ELBW infants: is sepsis increased? World J Pediatr.

2010;6(2):154-7.

\footnotetext{
REFERÊNCIAS

1. Pettit J, Wyckoff MM. Peripherally inserted central catheters: Neonatal Nurses. 2007.

2. Conselho Federal de Enfermagem. Resolução COFEN-

258/2001. Inserção de Cateter Periférico Central, pelos

Enfermeiros. Rio de Janeiro (Brasil); COFEN; 2001.

3. Dórea E, Castro TE, Costa P, Kimura AF, Santos FMG.
} 
6. Costa $\mathrm{P}$, Camargo PP, Bueno M, Kimura AF. Measuring pain in neonates during placement of central line catheter via peripheral insertion. Acta paul. enferm. 2010;23(1):35-40.

7. O'Grady NP, Alexander M, Dellinger EP, Gerberding JL, Heard SO, Maki DG, et al. Guidelines for the prevention of intravascular catheter-related infections. Centers for Disease Control and Prevention. MMWR Recomm Rep 2002;51(RR-10):129.

8. Hoang V, Sills J, Chandler, Busalani E, Clifton-Koeppel R, Modanlou HD. Percutaneously Inserted Central Catheter for Total Parenteral Nutrition in neonates: comlications rates related to upper versus lower extremity insertion. Pediatrics. 2008;121(5):1152-1159.

9. Levy I, Bendet M, Samra Z, Shalit I, Katz J. Infectious complications of peripherally inserted central venous catheters in children. Pediatr Infect Dis J. 2010;29(5):426-9.

10. O'Grady NP, Alexander M, Burns LA, Dellinger EP, Garland J, Heard SO, et al. Guidelines for the prevention of intravascular catheter-related infections. Clin Infect Dis 2011;52(9):e162:e193.

11. Curry S, Honeycutt M, Goins G, Gilliam C. CatheterAssociated Bloodstream Infections in the NICU: Getting to Zero. Neonatal Netw. 2009;28(3):151-5.

12. World alliance for patient safety. Forward Programme 20082009. WHO: Geneva, 2008.

13. Ragavan M, Gazula S, Yadav DK, Agarwala S, Srinivas M, Bajpai $M$, et al. Peripherally Inserted Central Venous Lines versus Central Lines in Surgical Newborns - A Comparison Indian J Pediatr. 2010;77(2):171-4.

14. Del Prato F, Di Matteo A, Messina F, Napolitano M. PICC: central venous access by the peripheral route. Medical-nursing aspects. Minerva Pediatr. 2010;62(3 Suppl 1):161-163.

15. Sarfraz MDA, Kapoor G. Peripherally Inserted Central Venous Catheters Are a Good Option for Prolonged Venous Access in Children with Cancer. Pediatr Blood Cancer. 2008; 51:251-255.

16. Montes SF, Teixeira JBA, Barbosa MH, Barichello E. Ocorrência de complicações relacionadas ao uso de Cateter Venoso Central de Inserção Periférica (PICC) em recémnascidos. Enfermería global. 2011;(24):10-18.

17. Camargo PP, Kimura AF, Toma E, Sunechiro MA. Initial placement of the peripherally inserted central catheter's tip in neonates. Rev Esc Enferm USP. 2008;42(4):723-8

18. Ormond V, Azevedo R, Gaiva M, Oliveira D. Perfil dos recémnascidos que utilizaram o cateter central de inserção periférica (CCIP) em um Hospital Universitário : estudo transversal. Online Brazilian journal of nursing. 2010;9(3):1-13.

19. Njere I, Islam S, Parish D, Kuna J, Keshtgar AS. Outcome of peripherally inserted central venous catheters in surgical and medical neonates. J Pediatr Surg. 2011;46(5):946-950 20. Marconi C, Cunha MLRS, Lyra JC, Bentlin MR, Batalha JEN, Sugizaki MF, et al. Usefulness of catheter tip culture in the diagnosis of neonatal infections. J. Pediatr. 2009;85(1):80-3.

Artigo recebido em 13/09/2011

Aprovado para publicação em 19/09/2012.

Artigo publicado em 31/03/2013. 